\title{
Logarithmic Interpolation Spaces between Quasi-Banach Spaces
}

\author{
Fernando Cobos, Luz M. Fernández-Cabrera, Antonio Manzano \\ and Antón Martínez
}

To the memory of Professor Miguel de Guzmán

\begin{abstract}
Let $A_{0}$ and $A_{1}$ be quasi-Banach spaces with $A_{0} \hookrightarrow A_{1}$. By means of a direct approach, we show that the interpolation spaces on $\left(A_{0}, A_{1}\right)$ generated by the function parameter $t^{\theta}(1+|\log t|)^{-b}$ can be expressed in terms of classical real interpolation spaces. Applications are given to Zygmund spaces $L_{p}(\log L)_{b}(\Omega)$, LorentzZygmund function spaces and operator spaces defined by using approximation numbers.

Keywords. Logarithmic interpolation spaces, real interpolation with a parameter function, Zygmund function spaces, Lorentz-Zygmund function spaces, operator spaces defined by using approximation numbers

Mathematics Subject Classification (2000). Primary 46B70, 46E30, 47B10, secondary $46 \mathrm{E} 35$
\end{abstract}

\section{Introduction}

In 1993, Triebel [31] studied the degree of compactness of the embedding from the (fractional) Sobolev space $H_{p}^{n / p}(\Omega)$ into the Orlicz space $L_{\infty}(\log L)_{b}(\Omega)$. Here $\Omega$ is a bounded domain in $\mathbb{R}^{n}$ with smooth boundary, $1<p<\infty$ and $b<\frac{1}{p}-1$. The investigation of this limiting case of the well known Sobolev embedding theorem goes back to Trudinger [33] and Strichartz [29]. The " $L_{p^{-}}$ counterpart" to the " $L_{\infty}$-case" considered by Triebel, was studied by Edmunds

F. Cobos: Departamento de Análisis Matemático, Facultad de Matemáticas, Universidad Complutense de Madrid, 28040 Madrid, Spain; cobos@mat.ucm.es

L. M. Fernández-Cabrera: Departamento de Matemática Aplicada, Escuela de Estadística, Universidad Complutense de Madrid, 28040 Madrid, Spain;

luz_fernandez-c@mat.ucm.es

A. Manzano: Departamento de Matemáticas y Computación, Escuela Politécnica Superior, Universidad de Burgos, 09001 Burgos, Spain; amanzano@ubu.es

A. Martínez: Departamento de Matemática Aplicada I, E.T.S. Ingenieros Industriales, Universidad de Vigo, 36200 Vigo, Spain; antonmar@uvigo.es

Authors have been supported in part by the Spanish Ministerio de Educación y Ciencia (MTM2004-01888). 
and Triebel [9, 10], where they determined the behaviour of entropy numbers of the embedding from $H_{n p /(n+s p)}^{s}(\Omega)$ into the Zygmund space $L_{p}(\log L)_{b}(\Omega)$.

A basic tool in the approach of Edmunds and Triebel is a representation theorem of Zygmund spaces $L_{p}(\log L)_{b}(\Omega)$ in terms of $L_{p}(\Omega)$ spaces. This characterization has intrinsic interest and has led Edmunds and Triebel to introduce in $[9,10]$ the so-called logarithmic Sobolev spaces, and to study in [11] the abstract construction that comes up replacing in the representation spaces $L_{p}(\Omega)$ by complex interpolation spaces. They called logarithmic interpolation spaces to the spaces defined in this way.

More recently, Triebel and the first two present authors [7] have investigated a similar construction but now based on the real interpolation spaces $\left(A_{0}, A_{1}\right)_{\theta, q}$. In this case, it turns out that logarithmic spaces coincide with those spaces obtained by real interpolation with the function parameter $t^{\theta}(1+|\log t|)^{-b}$. As a consequence they have established representation theorems for Zygmund spaces $L_{p}(\log L)_{b}(\Omega)$ in terms of Lorentz spaces $L_{r, s}(\Omega)$, and characterizations for Lorentz-Zygmund operator spaces $\mathcal{L}_{p, q, b}(H)$ in terms of Lorentz operator spaces $\mathcal{L}_{r, s}(H)$. Here $H$ is a Hilbert space.

The results of [7] refer to the Banach case. They do not apply to spaces $L_{p}(\log L)_{b}(\Omega)$ for $0<p<1$, and they do not cover the extension of $\mathcal{L}_{p, q, b}(H)$ to operator spaces on Banach spaces, because operator spaces defined in terms of approximation numbers are only quasi-Banach spaces, even if $1<p, q<\infty$. To accomplish these results one should study logarithmic interpolation spaces in the class of quasi-Banach spaces.

From the point of view of extrapolation theory, logarithmic spaces are special cases of the more general notion of "one-sided" $\Sigma^{(p)-}$ and $\delta^{(p)-}$ spaces in the sense of Karadzhov and Milman [19]. In that recent paper (see also [13]) it is given an extensive study of the $\Sigma^{(p)}$ and $\Delta^{(p)}$ methods of extrapolation, complementing the previous work of Jawerth and Milman [18, 22] which deals mainly with the $\Sigma^{(1)}$ and $\Delta^{(\infty)}$ methods. Since results of [19] work for quasiBanach spaces, one can apply them to derive results on abstract and concrete logarithmic interpolation spaces. In particular, Theorems 4.4 and 4.7 of [19] show that representation theorems for $L_{p}(\log L)_{b}(\Omega)$ in terms of spaces $L_{r, s}(\Omega)$ hold for the full range of parameters.

In this paper we study quasi-Banach logarithmic interpolation spaces by following a direct approach, based on ideas of [7]. We start by showing that logarithmic spaces generated by quasi-Banach couples coincide also with interpolation spaces obtained by using function parameters. Then we investigate the role of the scalar parameter $q$ of real interpolation in logarithmic spaces. The value of $q$ is the same for all real interpolation spaces that appear in the definition of logarithmic spaces (see Definition 2.1 below) and it coincides with the power of the summation over $j$ as well. This is a help for computations 
but it is also the reason why representation theorems of [7] for $L_{p}(\log L)_{b}(\Omega)$ are given in terms of Lorentz spaces, instead of the simpler Lebesgue spaces. We show here that the construction of logarithmic spaces is sufficiently flexible to allow certain changes of $q$ with the summing index $j$. As a consequence, applying the abstract results to Zygmund spaces $L_{p}(\log L)_{b}(\Omega)$, we derive representations that only require Lebesgue spaces and that work for $0<p<1$ as well.

Moreover, we apply the abstract results to spaces $\mathcal{L}_{p, q, b}(E, F)$, formed by all operators $T$ acting between the quasi-Banach spaces $E$ and $F$, whose approximation numbers $\left\{a_{m}(T)\right\}$ lie in the Lorentz-Zygmund sequence space $\ell_{p, q}(\log \ell)_{b}$ (see [5] and [6]). Spaces $\mathcal{L}_{p, q, b}(E, F)$ are the natural extension of $\mathcal{L}_{p, q, b}(H)$. Some results on bounded linear maps between spaces $\mathcal{L}_{p, q, b}(E, F)$ are also established. This kind of application is not considered in [19]. It is also not covered by the results of [13].

The organization of the paper is as follows. In Section 2 we study logarithmic interpolation spaces in the quasi-Banach setting. Section 3 deals with the applications to function spaces. Finally, in Section 4, we give the applications to operator spaces.

\section{Logarithmic interpolation spaces}

Let $A_{0}, A_{1}$ be quasi-Banach spaces with $A_{0} \hookrightarrow A_{1}$, where the notation $\hookrightarrow$ means continuous inclusion. The Peetre's $K$-functional and $J$-functional are defined by

$$
\begin{aligned}
K(t, a) & =K\left(t, a ; A_{0}, A_{1}\right) \\
& =\inf \left\{\left\|a_{0}\right\|_{A_{0}}+t\left\|a_{1}\right\|_{A_{1}}: a=a_{0}+a_{1}, a_{j} \in A_{j}\right\}, \quad t>0, a \in A_{1},
\end{aligned}
$$

and

$$
J(t, a)=J\left(t, a ; A_{0}, A_{1}\right)=\max \left\{\|a\|_{A_{0}}, t\|a\|_{A_{1}}\right\}, \quad t>0, a \in A_{0} .
$$

For $0<\theta<1$ and $0<q \leq \infty$, the real interpolation space $A_{\theta, q}=\left(A_{0}, A_{1}\right)_{\theta, q}$ is formed by all those elements $a \in A_{1}$ having a finite quasi-norm

$$
\|a\|_{A_{\theta, q}}= \begin{cases}\left(\int_{0}^{\infty}\left(t^{-\theta} K(t, a)\right)^{q} \frac{d t}{t}\right)^{\frac{1}{q}} & \text { if } 0<q<\infty \\ \sup _{t>0}\left\{t^{-\theta} K(t, a)\right\} & \text { if } q=\infty\end{cases}
$$

(see [4] and [30]). It is well known that the equivalence theorem still holds in the quasi-Banach setting (see [4, Theorem 3.11.3]), so $A_{\theta, q}$ can be equivalently realized as a $J$-space. 
Using that $A_{0} \hookrightarrow A_{1}$, it is not hard to check that $\|a\|_{A_{\theta, q}}$ is equivalent to any of the following quasi-norms:

$$
\left(\sum_{m=1}^{\infty} 2^{-\theta m q} K^{q}\left(2^{m}, a\right)\right)^{\frac{1}{q}}, \quad \inf \left\{\left(\sum_{m=1}^{\infty} 2^{-\theta m q} J^{q}\left(2^{m}, a_{m}\right)\right)^{\frac{1}{q}}: a=\sum_{m=1}^{\infty} a_{m}\right\}
$$

(with the usual modification if $q=\infty$ ), where the infimum is extended over all representations $a=\sum_{m=1}^{\infty} a_{m}$ (convergence in $A_{1}$ ), with $a_{m} \in A_{0}$ and $\left(\sum_{m=1}^{\infty} 2^{-\theta m q} J^{q}\left(2^{m}, a_{m}\right)\right)^{\frac{1}{q}}<\infty$. Constants in equivalences depend on $\theta$ and $q$, but if $\theta$ runs on a compact subset of $(0,1)$, say

$$
\theta \in\left\{\eta+2^{-j}: j \geq j_{0}\right\} \cup\left\{\eta-2^{-j}: j \geq j_{0}\right\} \cup\{\eta\}
$$

as it is the case in Definition 2.1, then it is possible to choose uniform constants for all those values of $\theta$. Subsequently, we denote any of these three quasi-norms by the symbol $\|\cdot\|_{A_{\theta, q}}$. This will cause no confusion.

Replacing in the definition of $A_{\theta, q}$ the function $t^{\theta}$ by a more general function parameter $\varrho(t)$ we obtain the spaces $A_{\varrho ; q}=\left(A_{0}, A_{1}\right)_{\varrho ; q}$ that have been studied in $[24,16,17]$ or $[25]$. We will mainly work here with the special function parameters

$$
\varrho(t)=\varrho_{\theta, b}(t)=t^{\theta}(1+|\log t|)^{-b}, \quad t>0,
$$

where $0<\theta<1$ and $\mathrm{b} \in \mathbb{R}$. Again, we have

$$
\begin{aligned}
& \|a\|_{A_{\varrho ; q}}=\left(\int_{0}^{\infty}\left(\frac{K(t, a)}{\varrho(t)}\right)^{q} \frac{d t}{t}\right)^{\frac{1}{q}} \\
& \sim\left(\sum_{m=1}^{\infty} \frac{K^{q}\left(2^{m}, a\right)}{\varrho^{q}\left(2^{m}\right)}\right)^{\frac{1}{q}} \\
& \sim \inf \left\{\left(\sum_{m=1}^{\infty} \frac{J^{q}\left(2^{m}, a_{m}\right)}{\varrho^{q}\left(2^{m}\right)}\right)^{\frac{1}{q}}: \begin{array}{c}
a=\sum_{m=1}^{\infty} a_{m} \text { with }\left\{a_{m}\right\} \subseteq A_{0} \text { and } \\
\left(\sum_{m=1}^{\infty} \frac{J^{q}\left(2^{m}, a_{m}\right)}{\varrho^{q}\left(2^{m}\right)}\right)^{\frac{1}{q}}<\infty
\end{array}\right\} .
\end{aligned}
$$

Here $\sim$ means equivalence of quasi-norms.

Since $A_{0} \hookrightarrow A_{1}$, we have for $0<p, q \leq \infty$

$$
\left(A_{0}, A_{1}\right)_{\mu, p} \hookrightarrow\left(A_{0}, A_{1}\right)_{\theta, q} \quad \text { if } 0<\mu<\theta<1
$$

(see [4, Theorem 3.4.1]). Let $A_{\theta+}=\bigcap_{\theta<\eta<1} A_{\eta, q}$, where $0<q \leq \infty$ and $0<\theta<1$. By (1), the space $A_{\theta+}$ is independent of $q$.

We shall now introduce logarithmic interpolation spaces in the quasi-Banach case by extending the definition of [7]. We denote by $\mathbb{N}$ the collection of all natural numbers. 
Definition 2.1. Let $A_{0}, A_{1}$ be quasi-Banach spaces with $A_{0} \hookrightarrow A_{1}$. Let $0<$ $\theta<1$ and let $j_{0}=j_{0}(\theta) \in \mathbb{N}$ such that, for all $j \in \mathbb{N}$ with $j \geq j_{0}$,

$$
\sigma_{j}=\theta+2^{-j}<1 \quad \text { and } \quad \lambda_{j}=\theta-2^{-j}>0 .
$$

Let $0<q \leq \infty$.

(i) Assume $b<0$. We let $A_{\theta, q}(\log A)_{b}$ denote the space of all $a \in A_{\theta+}$ which have a finite quasi-norm

$$
\|a\|_{A_{\theta, q}(\log A)_{b}}=\left(\sum_{j=j_{0}}^{\infty} 2^{j b q}\|a\|_{A_{\sigma_{j}, q}}^{q}\right)^{\frac{1}{q}}
$$

(with the usual modification if $q=\infty$ ).

(ii) Let $b>0$. The space $A_{\theta, q}(\log A)_{b}$ consists of all $a \in A_{1}$ which can be represented as

$$
a=\sum_{j=j_{0}}^{\infty} a_{j}, \quad \text { convergence in } A_{1}, \text { with } a_{j} \in A_{\lambda_{j}, q}
$$

such that $\left(\sum_{j=j_{0}}^{\infty} 2^{j b q}\left\|a_{j}\right\|_{A_{\lambda_{j}, q}}^{q}\right)^{\frac{1}{q}}<\infty$. We put

$$
\|a\|_{A_{\theta, q}(\log A)_{b}}=\inf \left\{\left(\sum_{j=j_{0}}^{\infty} 2^{j b q}\left\|a_{j}\right\|_{A_{\lambda_{j}, q}}^{q}\right)^{\frac{1}{q}}\right\}
$$

where the infimum is taken over all sequences $\left\{a_{j}\right\}$ satisfying (2).

(iii) If $b=0$, then $A_{\theta, q}(\log A)_{b}=A_{\theta, q}$.

Next we show that in the quasi-Banach setting the equality between logarithmic interpolation spaces and real interpolation spaces generated by function parameters $\varrho_{\theta, b}$ still holds. This result follows from [19, Theorems 4.2 and 4.6], because $A_{\theta, q}(\log A)_{b}$ can be realized as a $\delta^{(p)-}$ extrapolation space for $b<0$ and as a $\Sigma^{(p)-}$ space for $b>0$. However we prefer to give a direct and simpler proof, following the main lines of $[7$, Theorem 1$]$.

Theorem 2.2. Let $0<q \leq \infty, 0<\theta<1$ and $b \in \mathbb{R}$. Let $\varrho_{\theta, b}(t)=t^{\theta}(1+$ $|\log t|)^{-b}, t>0$. Then we have, with equivalent quasi-norms,

$$
A_{\theta, q}(\log A)_{b}=A_{\varrho_{\theta, b} ; q} .
$$

Proof. The proof of the case $b \leq 0$ goes through as in the Banach case (see [7, Theorem 1/Step 1]), because triangle inequality is not used there. To establish the case $b>0$, howewer, we have to modify the argument given in [7]. Assume therefore that $b>0$. Take any $a \in A_{\theta, q}(\log A)_{b}$ and suppose that $0<q<\infty$. Given any $\varepsilon>0$, we can find a representation $a=\sum_{j=j_{0}}^{\infty} a_{j}$ with $a_{j} \in A_{\lambda_{j}, q}$ and

$$
\sum_{j=j_{0}}^{\infty} 2^{j b q}\left\|a_{j}\right\|_{A_{\lambda_{j}, q}}^{q} \leq(1+\varepsilon)\|a\|_{A_{\theta, q}(\log A)_{b}}^{q} .
$$


Choose now decompositions $a_{j}=\sum_{m=1}^{\infty} a_{j}^{m}, j \geq j_{0}$, such that $\left\{a_{j}^{m}\right\} \subseteq A_{0}$ and

$$
\sum_{m=1}^{\infty} 2^{-m q\left(\theta-2^{-j}\right)} J^{q}\left(2^{m}, a_{j}^{m}\right) \leq(1+\varepsilon)\left\|a_{j}\right\|_{A_{\lambda_{j}, q}}^{q} .
$$

Let $c_{j}$ be the constant in the triangle inequality of $A_{j}(j=0,1)$, put $c=$ $\max \left\{c_{0}, c_{1}\right\}$ and define $r$ by the formula $(2 c)^{r}=2$. We can suppose that the $c_{j}$ are large, so that $r<q$. Let $\frac{1}{s}=\frac{1}{r}-\frac{1}{q}$. By Hölder's inequality, we have

$$
\begin{aligned}
& \left(\sum_{j=j_{0}}^{\infty} J\left(2^{m}, a_{j}^{m}\right)^{r}\right)^{\frac{1}{r}} \\
& \quad \leq\left(\sum_{j=j_{0}}^{\infty} 2^{-m q\left(\theta-2^{-j}\right)+j b q} J^{q}\left(2^{m}, a_{j}^{m}\right)\right)^{\frac{1}{q}}\left(\sum_{j=j_{0}}^{\infty} 2^{m s\left(\theta-2^{-j}\right)-j b s}\right)^{\frac{1}{s}} \\
& \quad \sim 2^{m \theta} m^{-b}\left(\sum_{j=j_{0}}^{\infty} 2^{-m q\left(\theta-2^{-j}\right)+j b q} J^{q}\left(2^{m}, a_{j}^{m}\right)\right)^{\frac{1}{q}},
\end{aligned}
$$

where the last equivalence follows by using that $b>0$ (see (33) in [7]).

The sum in (3) is finite as the argument below shows. Since the quasinorm $J\left(2^{m}, \cdot\right)$ is a $c$-norm, it follows from [4, Lemma 3.10.2] that $\sum_{j=j_{0}}^{\infty} a_{j}^{m}$ is convergent in $A_{0}$, say to $a^{m}$, with

$$
J\left(2^{m}, a^{m}\right) \leq C 2^{m \theta} m^{-b}\left(\sum_{j=j_{0}}^{\infty} 2^{-m q\left(\theta-2^{-j}\right)+j b q} J^{q}\left(2^{m}, a_{j}^{m}\right)\right)^{\frac{1}{q}} .
$$

Consequently, $a=\sum_{m=1}^{\infty} a^{m}$ with

$$
\begin{aligned}
\|a\|_{A_{\varrho ; q}}^{q} & \leq \sum_{m=1}^{\infty} \frac{J^{q}\left(2^{m}, a^{m}\right)}{\varrho_{\theta, b}^{q}\left(2^{m}\right)} \\
& \sim \sum_{m=1}^{\infty} 2^{-m \theta q} m^{b q} J^{q}\left(2^{m}, a^{m}\right) \\
& \leq C^{q} \sum_{j=j_{0}}^{\infty} 2^{j b q} \sum_{m=1}^{\infty} 2^{-m q\left(\theta-2^{-j}\right)} J^{q}\left(2^{m}, a_{j}^{m}\right) \\
& \leq C^{q}(1+\varepsilon) \sum_{j=j_{0}}^{\infty} 2^{j b q}\left\|a_{j}\right\|_{A_{\lambda_{j}, q}}^{q} \\
& \leq C^{q}(1+\varepsilon)^{2}\|a\|_{A_{\theta, q}(\log A)_{b}}^{q} .
\end{aligned}
$$

This implies that $A_{\theta, q}(\log A)_{b} \hookrightarrow A_{\varrho_{\theta, b} ; q}$. The case $q=\infty$ can be treated analogously. 
The converse embedding can be checked by using the same argument as in [7, Theorem 1/Step 2]. Suppose $q<\infty$. The proof when $q=\infty$ can be carried out in the same way. Let $a \in A_{\varrho_{\theta, b} ; q}$ and take any representation $a=\sum_{m=1}^{\infty} a_{m}$ with $\left\{a_{m}\right\} \subseteq A_{0}$ and $\sum_{m=1}^{\infty} \frac{J^{q}\left(2^{m}, a_{m}\right)}{\varrho_{\theta, b}^{q}\left(2^{m}\right)}<\infty$. Put

$$
a^{j}=\sum_{m=2^{j-j_{0}}}^{2^{j-j_{0}+1}-1} a_{m} \text { for } j \geq j_{0}
$$

Then we have $a^{j} \in A_{\lambda_{j}, q}, a=\sum_{j=j_{0}}^{\infty} a^{j}$ and

$$
\begin{aligned}
\|a\|_{A_{\theta, q}(\log A)_{b}}^{q} & \leq \sum_{j=j_{0}}^{\infty} 2^{j b q}\left\|a^{j}\right\|_{A_{\lambda_{j}, q}}^{q} \\
& \leq \sum_{j=j_{0}}^{\infty} 2^{j b q} \sum_{m=2^{j-j_{0}}}^{2^{j-j_{0}+1}-1} 2^{-m q\left(\theta-2^{-j}\right)} J^{q}\left(2^{m}, a_{m}\right) \\
& \sim \sum_{m=1}^{\infty} 2^{-m q \theta} m^{b q} J^{q}\left(2^{m}, a_{m}\right) .
\end{aligned}
$$

This yields that $A_{\varrho_{\theta, b} ; q} \hookrightarrow A_{\theta, q}(\log A)_{b}$ and finishes the proof.

In the proof of Theorem 2.2, it has been a help that summation over $j$ in Definition 2.1 is taken to the same power $q$ as in the spaces $A_{\sigma_{j}, q}$ and $A_{\lambda_{j}, q}$. Howewer, in applications to concrete couples we shall need that $q$ changes with $j$. Next we show that the choices of $q$ that we shall take later generate the same logarithmic interpolation spaces. More general results valid for $\Sigma^{(p)}$ and $\Delta^{(p)}$ extrapolation spaces can be found in [19, Theorems 2.13 and 3.4].

Theorem 2.3. Let $A_{0}, A_{1}$ be quasi-Banach spaces with $A_{0} \hookrightarrow A_{1}$. Let $0<\theta<1$ and let $j_{0}=j_{0}(\theta) \in \mathbb{N}$ such that, for all $j \in \mathbb{N}, j \geq j_{0}, \sigma_{j}=\theta+2^{-j}<1$. Let $b<0,0<q \leq \infty$ and $r>0$. Put $\frac{1}{s_{j}}=\frac{1}{q}+\frac{1}{r 2^{j}}, j \geq j_{0}$. Then $A_{\theta, q}(\log A)_{b}$ consists of all $a \in A_{\theta+}$ such that

$$
\|a\|_{*}=\left(\sum_{j=j_{0}}^{\infty} 2^{j b q}\|a\|_{A_{\sigma_{j}, s_{j}}}^{q}\right)^{\frac{1}{q}}<\infty
$$

Moreover, the quasi-norms $\|\cdot\|_{A_{\theta, q}(\log A)_{b}}$ and $\|\cdot\|_{*}$ are equivalent.

Proof. Since $s_{j}<q$ for any $j \geq j_{0}$, we have

$$
\|a\|_{A_{\sigma_{j}, q}}=\left(\sum_{m=1}^{\infty} 2^{-m q \sigma_{j}} K^{q}\left(2^{m}, a\right)\right)^{\frac{1}{q}} \leq\left(\sum_{m=1}^{\infty} 2^{-m s_{j} \sigma_{j}} K^{s_{j}}\left(2^{m}, a\right)\right)^{\frac{1}{s_{j}}}=\|a\|_{A_{\sigma_{j}, s_{j}}} .
$$


Hence, given any $a \in A_{\theta+}$, we get

$$
\|a\|_{A_{\theta, q}(\log A)_{b}} \leq\left(\sum_{j=j_{0}}^{\infty} 2^{j b q}\|a\|_{A_{\sigma_{j}, s_{j}}}^{q}\right)^{\frac{1}{q}}=\|a\|_{*} .
$$

On the other hand, we claim that there exists $M>0$ such that

$$
\|a\|_{A_{\sigma_{j}, s_{j}}} \leq M\|a\|_{A_{\sigma_{j+1}, q}} \text { for all } j \geq j_{0} .
$$

Indeed, using Hölder's inequality, we get

$$
\begin{aligned}
\|a\|_{A_{\sigma_{j}, s_{j}}} & =\left(\sum_{m=1}^{\infty} 2^{-m s_{j} \sigma_{j}} K^{s_{j}}\left(2^{m}, a\right)\right)^{\frac{1}{s_{j}}} \\
& \leq\left(\sum_{m=1}^{\infty} 2^{-m q \sigma_{j+1}} K^{q}\left(2^{m}, a\right)\right)^{\frac{1}{q}}\left(\sum_{m=1}^{\infty} 2^{m r 2^{j}\left(\sigma_{j+1}-\sigma_{j}\right)}\right)^{\frac{1}{r 2^{j}}} \\
& =\|a\|_{A_{\sigma_{j+1}, q}}\left(\sum_{m=1}^{\infty} 2^{-m r / 2}\right)^{\frac{1}{r 2^{j}}} \\
& \leq M\|a\|_{A_{\sigma_{j+1}, q}}
\end{aligned}
$$

Consequently, for any $a \in A_{\theta+}$, we derive $\|a\|_{*} \leq M 2^{-b}\|a\|_{A_{\theta, q}(\log A)_{b}}$.

The corresponding result for $b>0$ reads as follows.

Theorem 2.4. Let $A_{0}, A_{1}$ be quasi-Banach spaces with $A_{0} \hookrightarrow A_{1}$. Let $b>0$, $0<\theta<1,0<q<\infty, r>0$ and let $j_{0}=j_{0}(\theta) \in \mathbb{N}$ such that, for all $j \in \mathbb{N}$, $j \geq j_{0}, \lambda_{j}=\theta-2^{-j}>0$ and $\frac{1}{r_{j}}=\frac{1}{q}-\frac{1}{r 2^{j}}>0$. Then $A_{\theta, q}(\log A)_{b}$ is formed by all those $a \in A_{1}$ which can be represented as $a=\sum_{j=j_{0}}^{\infty} a_{j}$, convergence in $A_{1}$, with $a_{j} \in A_{\lambda_{j}, r_{j}}$ and $\left(\sum_{j=j_{0}}^{\infty} 2^{j b q}\left\|a_{j}\right\|_{A_{\lambda_{j}, r_{j}}}^{q}\right)^{\frac{1}{q}}<\infty$. Moreover,

$$
\|a\|_{* *}=\inf \left\{\left(\sum_{j=j_{0}}^{\infty} 2^{j b q}\left\|a_{j}\right\|_{A_{\lambda_{j}, r_{j}}}^{q}\right)^{\frac{1}{q}}\right\}
$$

is an equivalent quasi-norm in the space $A_{\theta, q}(\log A)_{b}$. Here the infimum is taken over all representations of the described type.

Proof. First we show that if $\sum_{j=j_{0}}^{\infty} 2^{j b q}\left\|a_{j}\right\|_{A_{\lambda_{j}, r_{j}}}^{q}<\infty$, then $\sum_{j=j_{0}}^{\infty} a_{j}$ is convergent in $A_{1}$. Let $c$ be the constant in the triangle inequality of $A_{1}$ and define $s$ by the formula $(2 c)^{s}=2$. We may assume that $s<q$. Put $\frac{1}{p}=\frac{1}{s}-\frac{1}{q}$. If $a \in A_{\lambda_{j}, r_{j}}$, we have

$$
\|a\|_{A_{1}} \sim K(2, a) \leq 2^{\theta}\|a\|_{A_{\lambda_{j}, r_{j}}} .
$$


Whence, applying Hölder's inequality, we obtain

$$
\left(\sum_{j=j_{0}}^{\infty}\left\|a_{j}\right\|_{A_{1}}^{s}\right)^{\frac{1}{s}} \leq 2^{\theta}\left(\sum_{j=j_{0}}^{\infty} 2^{j b q}\left\|a_{j}\right\|_{A_{\lambda_{j}, r_{j}}}^{q}\right)^{\frac{1}{q}}\left(\sum_{j=j_{0}}^{\infty} 2^{-j b p}\right)^{\frac{1}{p}}<\infty .
$$

This yields that $\sum_{j=j_{0}}^{\infty} a_{j}$ is convergent in $A_{1}$.

Since $q<r_{j}$, it follows that $\|a\|_{* *} \leq\|a\|_{A_{\theta, q}(\log A)_{b}}$. To establish the converse inequality, we proceed as in Theorem 2.3. For any $j \geq j_{0}$ and any $a \in A_{\lambda_{j}, r_{j}}$, we get

$$
\begin{aligned}
\|a\|_{A_{\lambda_{j+1}, q}} & =\left(\sum_{m=1}^{\infty} 2^{-m q \lambda_{j+1}} K^{q}\left(2^{m}, a\right)\right)^{\frac{1}{q}} \\
& \leq\left(\sum_{m=1}^{\infty} 2^{-m r_{j} \lambda_{j}} K^{r_{j}}\left(2^{m}, a\right)\right)^{\frac{1}{r_{j}}}\left(\sum_{m=1}^{\infty} 2^{m\left(\lambda_{j}-\lambda_{j+1}\right) r 2^{j}}\right)^{\frac{1}{r 2^{j}}} \\
& =\|a\|_{A_{\lambda_{j}, r_{j}}}\left(\sum_{m=1}^{\infty} 2^{-m r / 2}\right)^{\frac{1}{r 2^{j}}} \\
& \leq M\|a\|_{A_{\lambda_{j}, r_{j}}} .
\end{aligned}
$$

This implies that $\|a\|_{A_{\theta, q}(\log A)_{b}} \leq M 2^{b}\|a\|_{* *}$.

Remark 2.5. Let $\frac{1}{s_{j}^{*}}=\frac{1}{q}-\frac{1}{r 2^{j}}$ and $\frac{1}{r_{j}^{*}}=\frac{1}{q}+\frac{1}{r 2^{j}}$. It is easy to check that Theorem 2.3 still holds for $q<\infty$ if we replace $s_{j}$ by $s_{j}^{*}$. Similarly, Theorem 2.4 is also valid if we replace $r_{j}$ by $r_{j}^{*}$.

Logarithmic spaces in the case $\theta=0$ and $q=\infty$ will be also useful later. If $A_{0}, A_{1}$ are quasi-Banach spaces with $A_{0} \hookrightarrow A_{1}$ and $b<0$, we let $A_{0, \infty}(\log A)_{b}$ denote the space of all $a \in A_{0+}$ which have a finite quasi-norm

$$
\|a\|_{A_{0, \infty}(\log A)_{b}}=\sup _{j \geq 1}\left\{2^{j b}\|a\|_{A_{2^{-j}, \infty}}\right\} \text {. }
$$

Here

$$
\|a\|_{A_{2^{-j}, \infty}}=\sup _{m \geq 1}\left\{2^{-2^{-j} m} K\left(2^{m}, a\right)\right\} .
$$

We put $\varrho_{0, b}(t)=(1+|\log t|)^{-b}, t>0$, and we denote by $\left(A_{0}, A_{1}\right)_{\varrho_{0, b} ; \infty}$ the collection of all those $a \in A_{1}$ which have a finite quasi-norm

$$
\|a\|_{A_{\varrho_{0, b} ; \infty}}=\sup _{t \geq 1}\left\{\frac{K(t, a)}{\varrho_{0, b}(t)}\right\} \text {. }
$$

Theorem 2.6. Let $b<0$. Then we have, with equivalent quasi-norms,

$$
A_{0, \infty}(\log A)_{b}=A_{\varrho_{0, b} ; \infty} .
$$

Proof. The result follows by using the same arguments as in Theorem 2.2. 
Remark 2.7. Spaces $A_{\theta, q}(\log A)_{b}$ might be considered as a quantitative counterpart to the notion of inclusion indices relative to an interpolation scale (see [12]).

\section{Applications to function spaces}

In this section we specialize the abstract results of Section 2 to Lorentz-Zygmund function spaces.

Let $\Omega$ be a domain in $\mathbb{R}^{n}$ with finite Lebesgue measure $|\Omega|$. For $0<p<\infty$, $0<q \leq \infty$ and $b \in \mathbb{R}$, the Lorentz-Zygmund function space $L_{p, q}(\log L)_{b}(\Omega)$ is formed by all (equivalent classes of) Lebesgue-measurable functions $f$ on $\Omega$ which have a finite quasi-norm

$$
\|f\|_{L_{p, q}(\log L)_{b}(\Omega)}=\left(\int_{0}^{|\Omega|}\left[t^{\frac{1}{p}}(1+|\log t|)^{b} f^{*}(t)\right]^{q} \frac{d t}{t}\right)^{\frac{1}{q}}
$$

(with the obvious modification if $q=\infty$ ). Here $f^{*}$ is the non-increasing rearrangement of $f$

$$
f^{*}(t)=\inf \{s>0:|\{x \in \Omega:|f(x)|>s\}| \leq t\} .
$$

We refer to [2, 3] and [23] for properties of Lorentz-Zygmund function spaces. Note that if $p=q$, we get the Zygmund spaces $L_{p}(\log L)_{b}(\Omega)$. In particular, for $b=0$ we obtain the Lebesgue spaces $L_{p}(\Omega)$. The case $b=0$ and $p \neq q$ gives the Lorentz function spaces $L_{p, q}(\Omega)$.

The following result extends [10, Theorem 2.6.2/2] to the range $0<p<1$.

Corollary 3.1. Let $\Omega$ be a domain in $\mathbb{R}^{n}$ with finite Lebesgue measure. Let $0<p<\infty$ and let $j_{0}=j_{0}(p) \in \mathbb{N}$ such that, for all $j \in \mathbb{N}$ with $j \geq j_{0}$, $\frac{1}{p^{\lambda_{j}}}=\frac{1}{p}-\frac{1}{n 2^{j}}>0$. Put $\frac{1}{p^{\sigma_{j}}}=\frac{1}{p}+\frac{1}{n 2^{j}}$.

(i) Let $b<0$. Then $L_{p}(\log L)_{b}(\Omega)$ is the set of all Lebesgue-measurable functions $f$ on $\Omega$ such that

$$
\left(\sum_{j=j_{0}}^{\infty} 2^{j b p}\|f\|_{L_{p} \sigma_{j}(\Omega)}^{p}\right)^{\frac{1}{p}}<\infty
$$

Moreover, (4) defines an equivalent quasi-norm on $L_{p}(\log L)_{b}(\Omega)$.

(ii) Let $b>0$. Then $L_{p}(\log L)_{b}(\Omega)$ is the set of all Lebesgue-measurable functions $f$ on $\Omega$ which can be represented as

$$
f=\sum_{j=j_{0}}^{\infty} f_{j}, \quad f_{j} \in L_{p^{\lambda_{j}}}(\Omega)
$$


such that

$$
\left(\sum_{j=j_{0}}^{\infty} 2^{j b p}\left\|f_{j}\right\|_{L_{p^{\lambda_{j}}}(\Omega)}^{p}\right)^{\frac{1}{p}}<\infty .
$$

Moreover, the infimum over expression (6) taken over all representations (5), (6) is an equivalent quasi-norm on $L_{p}(\log L)_{b}(\Omega)$.

Proof. Take $0<r<p$ and let $\theta=\frac{r}{p}$. Consider the spaces $L_{\infty}(\Omega)$ and $L_{r}(\Omega)$. Since $|\Omega|<\infty$, we have $L_{\infty}(\Omega) \hookrightarrow L_{r}(\Omega)$. According to [4, Theorem 5.2.1],

$$
K\left(t, f ; L_{\infty}(\Omega), L_{r}(\Omega)\right) \sim t\left(\int_{0}^{t^{-r}}\left(f^{*}(s)\right)^{r} d s\right)^{\frac{1}{r}} .
$$

Hence, interpolating with $\varrho_{\theta, b}(t)=t^{\theta}(1+|\log t|)^{-b}, t>0$, we get

$$
\left(L_{\infty}(\Omega), L_{r}(\Omega)\right)_{\varrho_{\theta, b} ; p}=L_{p}(\log L)_{b}(\Omega)
$$

with equivalent quasi-norms.

$$
\begin{aligned}
& \text { Put } \frac{1}{p_{*}^{\sigma_{j}}}=\frac{1}{p}+\frac{1}{r 2^{j}} \text { and } \frac{1}{p_{*}^{\lambda_{j}}}=\frac{1}{p}-\frac{1}{r 2^{j}} \text {. Then } \\
& \qquad\left(L_{\infty}(\Omega), L_{r}(\Omega)\right)_{\theta+2^{-j}, p_{*}^{\sigma_{j}}}=L_{p_{*}} \sigma_{\sigma_{j}}(\Omega)
\end{aligned}
$$

and

$$
\left(L_{\infty}(\Omega), L_{r}(\Omega)\right)_{\theta-2^{-j}, p_{*}^{\lambda_{j}}}=L_{p_{*}^{\lambda_{j}}}(\Omega)
$$

with equivalence of quasi-norms where the constants do not depend on $j$. Whence, for $b<0$, it follows from Theorems 2.2 and 2.3 that $L_{p}(\log L)_{b}(\Omega)$ is the set of all measurable functions $f$ on $\Omega$ such that

$$
\left(\sum_{j=j_{0}}^{\infty} 2^{j b p}\|f\|_{L_{p_{*} \sigma_{j}}(\Omega)}^{p}\right)^{\frac{1}{p}}<\infty .
$$

On the other hand, for $b>0$, Theorems 2.2 and 2.4 imply that $L_{p}(\log L)_{b}(\Omega)$ consists of all measurable functions $f$ on $\Omega$ which can be represented as

$$
f=\sum_{j=j_{0}}^{\infty} f_{j}, \quad f_{j} \in L_{p_{*}^{\lambda_{j}}}(\Omega)
$$

such that

$$
\left(\sum_{j=j_{0}}^{\infty} 2^{j b p}\left\|f_{j}\right\|_{L_{p_{*}}(\Omega)}^{p}\right)^{\frac{1}{p}}<\infty .
$$

Finally, using Hölder's inequality and the fact that $|\Omega|<\infty$, it is not difficult to show that (8) and (4) are equivalent, and that the infimum over expression (9) is an equivalent quasi-norm to the one defined by (6). 
Part (i) in Corollary 3.1 was proved by Edmunds and Triebel in [10, Theorem 2.6.2/1] by direct calculations. Then, using duality, they derived part (ii) in Corollary 3.1 for $1 \leq p<\infty$ (see [10, Theorem 2.6.2/2]). Note that their technique does not allow to cover the case $0<p<1$, because in this range $\left(L_{p}(\Omega)\right)^{\prime}=\{0\}$.

If $p=\infty$, we can recover from the outcome for $\theta=0$ the following result due to Triebel [31].

Corollary 3.2. Let $\Omega$ be a domain in $\mathbb{R}^{n}$ with finite Lebesgue measure, let $b<0$ and let $p^{\sigma_{j}}=n 2^{j}$. Then $L_{\infty}(\log L)_{b}(\Omega)$ is the set of all Lebesgue-measurable functions $f$ on $\Omega$ such that

$$
\sup _{j \geq 1}\left\{2^{j b}\|f\|_{L_{p} \sigma_{j}(\Omega)}\right\}<\infty
$$

Moreover the expression in (10) defines an equivalent norm on $L_{\infty}(\log L)_{b}(\Omega)$.

Proof. Using (7) and Hardy's inequality (see [3, p. 246] or [2, Theorem 6.4]), we get

$$
\begin{aligned}
\|f\|_{\left(L_{\infty}(\Omega), L_{1}(\Omega)\right)_{\varrho_{0, b} ; \infty}} & =\sup _{t \geq 1}\left\{\frac{K\left(t, f ; L_{\infty}(\Omega), L_{1}(\Omega)\right)}{(1+|\log t|)^{-b}}\right\} \\
& =\sup _{t \geq 1}\left\{(1+|\log t|)^{b} t \int_{0}^{\frac{1}{t}} f^{*}(s) d s\right\} \\
& =\sup _{0<t \leq 1}\left\{(1+|\log t|)^{b} \frac{1}{t} \int_{0}^{t} f^{*}(s) d s\right\} \\
& \sim \sup _{0<t \leq 1}\left\{(1+|\log t|)^{b} f^{*}(t)\right\} \\
& =\|f\|_{L_{\infty}(\log L)_{b}(\Omega) .}
\end{aligned}
$$

On the other hand, we have

$$
\begin{aligned}
\|f\|_{\left(L_{\infty}(\Omega), L_{1}(\Omega)\right)_{2}-j, \infty} & =\sup _{m \geq 1}\left\{2^{-2^{-j} m} K\left(2^{m}, f\right)\right\} \\
& \sim \sup _{t>0}\left\{t^{1-2^{-j}} \int_{0}^{\frac{1}{t}} f^{*}(s) d s\right\} \\
& \sim \sup _{t>0}\left\{t^{2^{-j}} f^{*}(t)\right\},
\end{aligned}
$$

where the constants in the equivalences do not depend on $j$. Since $|\Omega|<\infty$, for all $j \in \mathbb{N}$ and any $f \in L_{p^{\sigma_{j}}}(\Omega)$, we have

$$
\sup _{t>0}\left\{t^{2^{-j}} f^{*}(t)\right\} \leq \max \left\{1,|\Omega|^{\frac{n-1}{2 n}}\right\}\|f\|_{L_{p} \sigma_{j}(\Omega)} .
$$


Moreover, if $n<2^{j_{0}}$, then we can find $M>0$ such that for all $j \geq j_{0}$ and any $f \in L_{2^{j+j_{0}, \infty}}(\Omega)$, we get

$$
\|f\|_{L_{p} \sigma_{j}(\Omega)} \leq M \sup _{t>0}\left\{t^{2^{-\left(j+j_{0}\right)}} f^{*}(t)\right\}
$$

Now the result follows from Theorem 2.6.

Next we show that [7, Corollary 2], holds for the full range of parameters. This result is due to Karadzhov and Milman [19, Theorems 4.4 and 4.7].

Corollary 3.3. Let $\Omega$ be a domain in $\mathbb{R}^{n}$ with finite Lebesgue measure. Let $0<p<\infty, 0<q \leq \infty$ and let $j_{0}=j_{0}(p) \in \mathbb{N}$ such that, for all $j \in \mathbb{N}$ with $j \geq j_{0}, \frac{1}{p^{\nu_{j}}}=\frac{1}{p}-2^{-j}>0$. Put $\frac{1}{p^{\mu_{j}}}=\frac{1}{p}+2^{-j}$.

(i) Let $b<0$. Then $L_{p, q}(\log L)_{b}(\Omega)$ is the set of all measurable functions $f$ on $\Omega$ such that

$$
\left(\sum_{j=j_{0}}^{\infty} 2^{j b q}\|f\|_{L_{p^{\mu} \mu_{j}, q}(\Omega)}^{q}\right)^{\frac{1}{q}}<\infty
$$

(equivalent norms).

(ii) Let $b>0$. Then $L_{p, q}(\log L)_{b}(\Omega)$ is the set of all measurable functions $f$ on $\Omega$ which can be represented as

$$
f=\sum_{j=j_{0}}^{\infty} f_{j}, \quad f_{j} \in L_{p^{\nu_{j}, q}}(\Omega)
$$

such that

$$
\left(\sum_{j=j_{0}}^{\infty} 2^{j b q}\left\|f_{j}\right\|_{L_{p^{\nu}, q}(\Omega)}^{q}\right)^{\frac{1}{q}}<\infty .
$$

The infimum of the expression in (12) taken over all admissible representations (11), (12) is an equivalent quasi-norm in $L_{p, q}(\log L)_{b}(\Omega)$.

Proof. The argument is similar to the one in the proof of Corollary 3.1. Take $0<r<\min \{1, p, q\}$ and let $\theta=\frac{r}{p}$. Using (7), we have

$$
\left(L_{\infty}(\Omega), L_{r}(\Omega)\right)_{\varrho_{\theta, b} ; q}=L_{p, q}(\log L)_{b}(\Omega) .
$$

Put $\sigma_{j}=\theta+2^{-j}, \lambda_{j}=\theta-2^{-j}, \frac{1}{p^{\eta_{j}}}=\frac{1}{p}+\frac{1}{r 2^{j}}$ and $\frac{1}{p^{\tau_{j}}}=\frac{1}{p}-\frac{1}{r 2^{j}}$. Then

$$
\left(L_{\infty}(\Omega), L_{r}(\Omega)\right)_{\sigma_{j}, q}=L_{p^{\eta_{j}, q}}(\Omega) \quad \text { and } \quad\left(L_{\infty}(\Omega), L_{r}(\Omega)\right)_{\lambda_{j}, q}=L_{p^{\tau_{j}, q}}(\Omega)
$$


with equivalence of quasi-norms where the constants are independent of $j$. Since $\frac{1}{p^{\eta_{j}}}-\frac{1}{p^{\mu_{j}}}=\frac{1-r}{r 2^{j}} \rightarrow 0$ as $j \rightarrow \infty$, we have

$$
\begin{aligned}
\|f\|_{L_{p^{\eta_{j}, q}}(\Omega)} & =\left(\int_{0}^{|\Omega|}\left(t^{\frac{1}{p^{\eta_{j}}}} f^{*}(t)\right)^{q} \frac{d t}{t}\right)^{\frac{1}{q}} \\
& \leq \sup _{0<t<|\Omega|}\left\{t^{\frac{1-r}{r^{j} j}}\right\}\left(\int_{0}^{|\Omega|}\left(t^{\frac{1}{p^{\mu_{j}}}} f^{*}(t)\right)^{q} \frac{d t}{t}\right)^{\frac{1}{q}} \\
& \leq|\Omega|^{\frac{1-r}{r^{j} j}}\|f\|_{L_{p^{\mu}} \mu_{, q}(\Omega)} \\
& \leq M\|f\|_{L_{p^{\mu}} \mu_{q}(\Omega) .}
\end{aligned}
$$

Similarly $\|f\|_{L_{p^{\nu_{j}, q}}(\Omega)} \leq M\|f\|_{L_{p^{\tau_{j}, q}}(\Omega)}$. Let $j_{1}>j_{0}$ with $1<r 2^{j_{1}}$. Since

$$
\frac{1}{p^{\mu_{j}}}-\frac{1}{p^{\eta_{j_{1}+j}}}=\frac{1}{p^{\tau_{j_{1}+j}}}-\frac{1}{p^{\nu_{j}}}=\frac{r 2^{j_{1}}-1}{r 2^{j_{1}+j}} \rightarrow 0 \quad \text { as } \quad j \rightarrow \infty,
$$

we derive

$$
\begin{aligned}
& \|f\|_{L_{p^{\mu} \mu_{j}, q}(\Omega)} \leq M_{1}\|f\|_{L_{p} \eta_{j_{1}+j, q}(\Omega)} \\
& \|f\|_{L_{p} \tau_{j_{1}+j, q}(\Omega)} \leq M_{1}\|f\|_{L_{p} \nu_{j, q}}(\Omega) \text {. }
\end{aligned}
$$

Applying Theorem 2.2 to the couple $\left(L_{\infty}(\Omega), L_{r}(\Omega)\right)$, we obtain a representation of $L_{p, q}(\log L)_{b}(\Omega)$ in terms of the Lorentz spaces $L_{p^{\eta_{j}, q}}(\Omega)$ if $b<0$, and in terms of $L_{p^{\tau_{j}, q}}(\Omega)$ if $b>0$. Then the result follows with the aid of the relationships between spaces $L_{p^{\eta_{j}, q}}(\Omega)$ and $L_{p^{\mu_{j}, q}}(\Omega)$, and between spaces $L_{p^{\tau_{j}, q}}(\Omega)$ and $L_{p^{\nu_{j}, q}}(\Omega)$, that we have shown before.

Remark 3.4. Other kind of decompositions for Lorentz-Zygmund function spaces can be found in $[8,3.4 .4]$ and the references given there.

Remark 3.5. As we have said in the Introduction, Corollary 3.1 is the basic tool for the estimates on entropy numbers derived in [9]. Another kind of applications can be found in the book by Edmunds and Triebel [10, Remark 5, pp. 74-75]. It refers to the Hardy-Littlewood maximal function

$$
(M f)(x)=\sup _{x \in Q} \frac{1}{|Q|} \int_{Q}|f(y)| d y,
$$

where the supremum is taken over all cubes $Q$ containing $x$ and with sides parallel to the coordinate axes. A classical result of Hardy and Littlewood says that if $f \in L_{1}(\log L)_{1}(\Omega)$, then $M f \in L_{1}(\Omega)$. This assertion can be extended to $L_{1}(\log L)_{1+b}(\Omega)$ with $b \geq 0$. Indeed, see [28, p. 23] or [2, Theorem 3.4], it holds

$$
\|M f\|_{L_{1}(\log L)_{b}(\Omega)} \leq c\|f\|_{L_{1}(\log L)_{b+1}(\Omega)} .
$$


In $[10$, p. 75$]$ one can find a simple proof of (13) by using Corollary 3.1.

Next consider the related operator

$$
M_{r} f=\left[M\left(|f|^{r}\right)\right]^{\frac{1}{r}}
$$

where $0<r<1$. This operator is useful in several situations (see, for example, $[32$, pp. 78,108$])$. Inequality (13) yields that

$$
M_{r}: L_{r}(\log L)_{b+1 / r}(\Omega) \longrightarrow L_{r}(\log L)_{b}(\Omega)
$$

is bounded. Indeed,

$$
\begin{aligned}
\left\|M_{r} f\right\|_{L_{r}(\log L)_{b}(\Omega)} & =\left(\int_{0}^{|\Omega|} t(1+|\log t|)^{b r} M\left(|f|^{r}\right)^{*}(t) \frac{d t}{t}\right)^{\frac{1}{r}} \\
& \leq c\left\||f|^{r}\right\|_{L_{1}(\log L)_{b r+1}(\Omega)}^{\frac{1}{r}} \\
& =c\left(\int_{0}^{|\Omega|}\left(t^{\frac{1}{r}}(1+|\log t|)^{b+\frac{1}{r}} f^{*}(t)\right)^{r} \frac{d t}{t}\right)^{\frac{1}{r}} \\
& =c\|f\|_{L_{r}(\log L)_{b+\frac{1}{r}}(\Omega)} .
\end{aligned}
$$

\section{Applications to operator spaces}

In this final section we apply the abstract results of Section 2 to operator spaces defined in terms of approximation numbers.

Let $E, F$ be quasi-Banach spaces and let $\mathcal{L}(E, F)$ be the quasi-Banach space of all bounded linear operators acting from $E$ into $F$. For $k \in \mathbb{N}$, the $k$-th approximation number $a_{k}(T)$ of $T$ is defined by

$$
a_{k}(T)=\inf \{\|T-R\|: R \in \mathcal{L}(E, F) \text { with } \operatorname{rank} R<k\} .
$$

Let $c$ be the constant in the triangle inequality of $F$ and let $s$ be defined by the equation $(2 c)^{s}=2$. It is easy to check that for $S, T \in \mathcal{L}(E, F)$ and $k, m \in \mathbb{N}$, it holds

$$
a_{k+m-1}^{s}(S+T) \leq a_{k}^{s}(S)+a_{m}^{s}(T) .
$$

For $0<p<\infty, 0<q \leq \infty$ and $b \in \mathbb{R}$, we define the Lorentz-Zygmund operator spaces $\mathcal{L}_{p, q, b}(E, F)$ as the collection of all those $T \in \mathcal{L}(E, F)$ having a finite quasi-norm

$$
\|T\|_{p, q, b}=\left(\sum_{m=1}^{\infty}\left(m^{\frac{1}{p}}(1+\log m)^{b} a_{m}(T)\right)^{q} m^{-1}\right)^{\frac{1}{q}}
$$


(with the usual modification if $q=\infty$ ). In Banach spaces these operator spaces have been studied in [5] and [6]. For $b=0$, we get the Lorentz operator spaces $\left(\mathcal{L}_{p, q}(E, F),\|\cdot\|_{p, q}\right)$ (see [21] and [27]). The special case $b=0$ and $p=q$ gives the spaces $\left(\mathcal{L}_{p}(E, F),\|\cdot\|_{p}\right)$, which are the analogues of the Schatten $p$-classes for approximation numbers (see [14] and [26]).

Approximation numbers coincide with singular numbers for operators in Hilbert space $H$. If $E=F=H, 1<p<\infty, 1 \leq q \leq \infty$ and $b \in \mathbb{R}$, then the functional obtained from (15) replacing $a_{m}(T)$ by $m^{-1} \sum_{j=1}^{m} a_{j}(T)$ is a norm, equivalent to $\|\cdot\|_{p, q, b}$. The resulting Banach space was denoted by $\mathcal{L}_{p, q, b}(H)$ in [7], where it has been shown a representation theorem for $\mathcal{L}_{p, q, b}(H)$ in terms of spaces $\mathcal{L}_{p, q}(H)$. Next we establish the corresponding results for operators spaces in quasi-Banach spaces. We start with a result on the $K$-functional. In the Banach case, this was proved by König [20, Proposition 1].

Given two non-negative functions (or two sequences) $u(t), v(t)$, we write $u(t) \lesssim v(t)$ if there is a positive constant $c$ such that $u(t) \leq c v(t)$ for all $t$. The equivalence $u(t) \sim v(t)$ holds if $u(t) \lesssim v(t)$ and $v(t) \lesssim u(t)$.

Lemma 4.1. Let $E, F$ be quasi-Banach spaces and let $0<r<\infty$. Then

$$
K\left(t, T ; \mathcal{L}_{r}(E, F), \mathcal{L}(E, F)\right) \sim K\left(t,\left\{a_{m}(T)\right\} ; \ell_{r}, \ell_{\infty}\right)
$$

Proof. It $t \leq 1$, we have

$$
K\left(t, T ; \mathcal{L}_{r}(E, F), \mathcal{L}(E, F)\right) \sim t\|T\|=t\left\|\left\{a_{m}(T)\right\}\right\|_{\ell_{\infty}}=K\left(t,\left\{a_{m}(T)\right\} ; \ell_{r}, \ell_{\infty}\right) .
$$

Suppose $t>1$. Choose $T_{t} \in \mathcal{L}(E, F)$ such that $\operatorname{rank}\left(T_{t}\right)<\left[t^{r}\right]$ and $\left\|T-T_{t}\right\| \leq 2 a_{\left[t^{r}\right]}(T)$. Here [ $\left.\cdot\right]$ is the greatest integer function. If $m<\left[t^{r}\right]$, it follows from (14) that

$$
a_{m}^{s}\left(T_{t}\right) \leq a_{m}^{s}(T)+\left\|T-T_{t}\right\|^{s} \leq\left(1+2^{s}\right) a_{m}^{s}(T) .
$$

If $m \geq\left[t^{r}\right]$, then $a_{m}\left(T_{t}\right)=0$. Whence

$$
\begin{aligned}
K\left(t, T ; \mathcal{L}_{r}(E, F), \mathcal{L}(E, F)\right) & \leq\left\|T_{t}\right\|_{r}+t\left\|T-T_{t}\right\| \\
& \leq c_{1}\left(\sum_{m=1}^{\left[t^{r}\right]} a_{m}^{r}(T)\right)^{\frac{1}{r}}+2 t a_{\left[t^{r}\right]}(T) \\
& \leq c_{1}\left(\sum_{m=1}^{\left[t^{r}\right]} a_{m}^{r}(T)\right)^{\frac{1}{r}}+c_{2}\left(\left[t^{r}\right] a_{\left[t^{r}\right]}^{r}(T)\right)^{\frac{1}{r}} \\
& \leq c_{3}\left(\sum_{m=1}^{\left[t^{r}\right]} a_{m}^{r}(T)\right)^{\frac{1}{r}} .
\end{aligned}
$$


We claim that

$$
K\left(t, T ; \mathcal{L}_{r}(E, F), \mathcal{L}(E, F)\right) \sim\left(\sum_{m=1}^{\left[t^{r}\right]} a_{m}^{r}(T)\right)^{\frac{1}{r}}
$$

Indeed, take any $S \in \mathcal{L}_{r}(E, F)$ and let $s$ be again the number appearing in (14). Without loss of generality we may assume that $s<r$. Using (14) and Minkowski's inequality we obtain

$$
\begin{aligned}
\left(\sum_{m=1}^{\left[t^{r}\right]} a_{m}^{r}(T)\right)^{\frac{1}{r}} & \sim\left(\sum_{m=1}^{\left[t^{r}\right]} a_{2 m-1}^{r}(T)\right)^{\frac{1}{r}} \\
& \leq\left(\sum_{m=1}^{\left[t^{r}\right]}\left(a_{m}^{s}(S)+a_{m}^{s}(T-S)\right)^{\frac{r}{s}}\right)^{\frac{1}{r}} \\
& \leq\left(\left(\sum_{m=1}^{\left[t^{r}\right]} a_{m}^{r}(S)\right)^{\frac{s}{r}}+\left(\sum_{m=1}^{\left[t^{r}\right]} a_{m}^{r}(T-S)\right)^{\frac{s}{r}}\right)^{\frac{1}{s}} \\
& \leq c_{4}\left(\left(\sum_{m=1}^{\left[t^{r}\right]} a_{m}^{r}(S)\right)^{\frac{1}{r}}+t\|T-S\|\right) \\
& \leq c_{4}\left(\|S\|_{r}+t\|T-S\|\right) .
\end{aligned}
$$

This yields (16). Now the result follows by using [4, Theorem 5.2.1].

Let $0<p<\infty, 0<q \leq \infty$ and $b \in \mathbb{R}$. Take $0<r<p$ and put $\theta=1-\left(\frac{r}{p}\right)$. The quasi-Banach space $\mathcal{L}_{r}(E, F)$ is continuously embedded in $\mathcal{L}(E, F)$. By Lemma 4.1 and a similar argument as in [5, Theorem 5.2] we get

$$
\left(\mathcal{L}_{r}(E, F), \mathcal{L}(E, F)\right)_{\varrho \theta, b ; q}=\mathcal{L}_{p, q, b}(E, F)
$$

Now we can establish

Corollary 4.2. Let $E$ and $F$ be quasi-Banach spaces. Let $0<p<\infty, 0<q \leq$ $\infty$ and let $j_{0}=j_{0}(p) \in \mathbb{N}$ such that, for all $j \in \mathbb{N}$ with $j \geq j_{0}, \frac{1}{p^{j}}=\frac{1}{p}-\frac{1}{2^{j}}>0$. Put $\frac{1}{p^{\mu_{j}}}=\frac{1}{p}+\frac{1}{2^{j}}$.

(i) Let $b<0$. Then $\mathcal{L}_{p, q, b}(E, F)$ is the set of all $T \in \mathcal{L}(E, F)$ such that

$$
\left(\sum_{j=j_{0}}^{\infty} 2^{j b q}\|T\|_{p^{\nu_{j}, q}}^{q}\right)^{\frac{1}{q}}<\infty
$$

(equivalent quasi-norms). 
(ii) Let $b>0$. Then $\mathcal{L}_{p, q, b}(E, F)$ consists of all $T \in \mathcal{L}(E, F)$ which can be represented as $T=\sum_{j=j_{0}}^{\infty} T_{j}$ with $T_{j} \in \mathcal{L}_{p^{\mu_{j}, q}}(E, F)$ such that

$$
\left(\sum_{j=j_{0}}^{\infty} 2^{j b q}\left\|T_{j}\right\|_{p^{\mu_{j}, q}}^{q}\right)^{\frac{1}{q}}<\infty .
$$

Furthermore, the infimum over expression (17) is an equivalent quasinorm in $\mathcal{L}_{p, q, b}(E, F)$.

Proof. Take $0<r<p$. Put $\theta=1-\left(\frac{r}{p}\right), \sigma_{j}=\theta+2^{-j}, \lambda_{j}=\theta-2^{-j}$, and let $p_{*}^{\nu_{j}}, p_{*}^{\mu_{j}}$ be the numbers defined by $\frac{1}{p_{*}^{\nu_{j}}}=\frac{1}{p}-\frac{1}{r 2^{j}}, \frac{1}{p_{*}^{\mu_{j}}}=\frac{1}{p}+\frac{1}{r 2^{j}}$. We have

$$
\left(\mathcal{L}_{r}(E, F), \mathcal{L}(E, F)\right)_{\sigma_{j}, q}=\mathcal{L}_{p_{*}{ }^{\nu_{j}}, q},\left(\mathcal{L}_{r}(E, F), \mathcal{L}(E, F)\right)_{\lambda_{j}, q}=\mathcal{L}_{p_{*}{ }^{\mu}, q}
$$

with equivalence of quasi-norms where the constants do not depend on $j$. By Theorem 2.2, the space $\mathcal{L}_{p, q, b}(E, F)$ can be represented in terms of spaces $\mathcal{L}_{p_{*}{ }_{j}, q}(E, F)$ when $b<0$, and in terms of spaces $\mathcal{L}_{p_{*}{ }_{j}, q}(E, F)$ if $b>0$. Then the result follows by comparing $\mathcal{L}_{p_{*}^{\nu_{j}}, q}(E, F)$ with $\mathcal{L}_{p^{\nu_{j}, q}}^{\nu^{\nu_{j}}}(E, F)$ and $\mathcal{L}_{p_{*}{ }_{\mu_{j}}, q}(E, F)$ with $\mathcal{L}_{p^{\mu_{j}}, q}(E, F)$.

If $p=q$, we can derive representations in terms of the simpler spaces $\mathcal{L}_{r}(E, F)$ :

Corollary 4.3. Let $E, F$ be quasi-Banach spaces. Let $0<p<\infty$ and let $j_{0}=j_{0}(p) \in \mathbb{N}$ such that, for all $j \in \mathbb{N}$ with $j \geq j_{0}, \frac{1}{p^{j} j}=\frac{1}{p}-2^{-j}>0$. Put $\frac{1}{p^{\mu_{j}}}=\frac{1}{p}+2^{-j}$.

(i) Let $b<0$. Then $\mathcal{L}_{p, p, b}(E, F)$ is the set of all $T \in \mathcal{L}(E, F)$ such that

$$
\left(\sum_{j=j_{0}}^{\infty} 2^{j b p}\|T\|_{p^{\nu_{j}}}^{p}\right)^{\frac{1}{p}}<\infty
$$

(equivalent quasi-norms).

(ii) Let $b>0$. Then $\mathcal{L}_{p, p, b}(E, F)$ is the set of all operators $T \in \mathcal{L}(E, F)$ which can be represented as $T=\sum_{j=j_{0}}^{\infty} T_{j}$ with $T_{j} \in \mathcal{L}_{p^{\mu_{j}}}(E, F)$ such that

$$
\left(\sum_{j=j_{0}}^{\infty} 2^{j b p}\left\|T_{j}\right\|_{p^{\mu_{j}}}^{p}\right)^{\frac{1}{p}}<\infty .
$$

Moreover, the infimum over expression (18) is an equivalent quasi-norm in $\mathcal{L}_{p, p, b}(E, F)$.

Proof. The result is a consequence of Theorems 2.2, 2.3, 2.4 and Remark 2.5. 
Next we deal with bounded linear maps between operator spaces. The corresponding result to (13) reads as follows:

Theorem 4.4. Let $E$ and $F$ be quasi-Banach spaces and let $\mathcal{F}$ be a bounded linear operator from $\mathcal{L}_{p}(E, F)$ into $\mathcal{L}_{p}(E, F)$ for $1<p \leq 2$. If $b<0$ and

$$
\|\mathcal{F}\|_{\mathcal{L}_{p}(E, F), \mathcal{L}_{p}(E, F)} \lesssim(p-1)^{-1} \quad \text { as } \quad p \downarrow 1,
$$

then $\mathcal{F}$ is bounded from $\mathcal{L}_{1,1, b}(E, F)$ into $\mathcal{L}_{1,1, b-1}(E, F)$.

Proof. According to Corollary $4.3 /(\mathrm{i})$ with $p=1$, we have

$$
\frac{1}{p^{\nu_{j}}}=1-\frac{1}{2^{j}} \quad \text { or } \quad\left(p^{\nu_{j}}-1\right) \sim 2^{-j} .
$$

Whence, for any $T \in \mathcal{L}_{1,1, b}(E, F)$, using the information on $\mathcal{F}$ and Corollary $4.3 /(\mathrm{i})$, we derive

$$
\|\mathcal{F} T\|_{1,1, b-1} \sim \sum_{j=j_{0}}^{\infty} 2^{j(b-1)}\|\mathcal{F} T\|_{p^{\nu_{j}}} \lesssim \sum_{j=j_{0}}^{\infty} 2^{j b}\|T\|_{p^{\nu_{j}}} \sim\|T\|_{1,1, b} .
$$

We finish the paper with a result for the case $b=0$. We define the space $\mathcal{L}_{\mathcal{M}}(E, F)$ as the collection of all those $T \in \mathcal{L}(E, F)$ having a finite quasi-norm

$$
\|T\|_{\mathcal{M}}=\sup _{m \geq 1}\left\{\frac{\sum_{j=1}^{m} a_{j}(T)}{1+\log m}\right\} .
$$

Clearly, $\mathcal{L}_{1,1,-1}(E, F) \subseteq \mathcal{L}_{\mathcal{M}}(E, F) \subseteq \mathcal{L}_{1, \infty,-1}(E, F)$, and in general the inclusions are strict. For example, if $a_{m}(T) \sim 1 / m$, then $T \in \mathcal{L}_{\mathcal{M}}(E, F)$ but $T \notin \mathcal{L}_{1,1,-1}(E, F)$. If $a_{m}(T) \sim \frac{1}{m}(1+\log m)$, then $T \in \mathcal{L}_{1, \infty,-1}(E, F)$, but $T \notin \mathcal{L}_{\mathcal{M}}(E, F)$.

For operators in Hilbert space $H$, the space $\mathcal{L}_{\mathcal{M}}(H)$ is referred in the literature as one of Macaev ideals (see [15] or [1]).

Lemma 4.5. Let $E, F$ be quasi-Banach spaces. Then we have, with equivalent quasi-norms,

$$
\left(\mathcal{L}_{1}(E, F), \mathcal{L}(E, F)\right)_{\varrho_{0,-1} ; \infty}=\mathcal{L}_{\mathcal{M}}(E, F) .
$$

Proof. Using Lemma 4.1, we get

$$
\begin{aligned}
\|T\|_{\varrho_{0,-1} ; \infty} & =\sup _{t \geq 1}\left\{\frac{K(t, T)}{1+|\log t|}\right\} \\
& \sim \sup _{m \geq 1}\left\{\frac{K(m, T)}{1+\log m}\right\} \\
& \sim \sup _{m \geq 1}\left\{\frac{\sum_{j=1}^{m} a_{j}(T)}{1+\log m}\right\} \\
& =\|T\|_{\mathcal{M}} .
\end{aligned}
$$


In the Hilbert case, interpolation properties of the Macaev ideal can be found in [1] and [22].

Theorem 4.6. Let $E$ and $F$ be quasi-Banach spaces and let $\mathcal{F}$ be a bounded linear operator from $\mathcal{L}_{p}(E, F)$ into $\mathcal{L}_{p}(E, F)$ for $1<p \leq 2$. If

$$
\|\mathscr{F}\|_{\mathcal{L}_{p}(E, F), \mathcal{L}_{p}(E, F)} \lesssim(p-1)^{-1} \quad \text { as } \quad p \downarrow 1,
$$

then $\mathcal{F}$ is bounded from $\mathcal{L}_{1}(E, F)$ into $\mathcal{L}_{\mathcal{M}}(E, F)$.

Proof. Let $p_{j}=\left(1-2^{-j}\right)^{-1}$. According to Theorem 2.6 and Lemma 4.5, we have

$$
\|T\|_{\mathcal{M}} \sim \sup _{j \geq 1}\left\{2^{-j}\|T\|_{p_{j}, \infty}^{*}\right\}
$$

where \|\|$_{p_{j}, \infty}^{*}$ is the norm in $\mathcal{L}_{p_{j}, \infty}(E, F)$ obtained by real interpolation on the couple $\left(\mathcal{L}_{1}(E, F), \mathcal{L}(E, F)\right)$ with parameters $2^{-j}$ and $\infty$. That is

$$
\|T\|_{p_{j}, \infty}^{*}=\sup _{m \geq 1}\left\{2^{-2^{-j} m} \sum_{k=1}^{2^{m}} a_{k}(T)\right\}
$$

This norm satisfies that $\|T\|_{p_{j}, \infty}^{*} \leq\|T\|_{p_{j}}$ for all $T \in \mathcal{L}_{p_{j}}(E, F)$. Indeed, using Hölder's inequality, we obtain

$$
2^{-2^{-j} m} \sum_{k=1}^{2^{m}} a_{k}(T) \leq 2^{-2^{-j} m}\left(\sum_{k=1}^{2^{m}} a_{k}^{p_{j}}(T)\right)^{\frac{1}{p_{j}}} 2^{m\left(1-\frac{1}{p_{j}}\right)} \leq\|T\|_{p_{j}} .
$$

Therefore, for any $T \in \mathcal{L}_{1}(E, F)$, we derive

$$
\|\mathcal{F} T\|_{\mathcal{M}} \sim \sup _{j \geq 1}\left\{2^{-j}\|\mathcal{F} T\|_{p_{j}, \infty}^{*}\right\} \leq \sup _{j \geq 1}\left\{2^{-j}\|\mathcal{F} T\|_{p_{j}}\right\} \lesssim \sup _{j \geq 1}\left\{\|T\|_{p_{j}}\right\} \leq\|T\|_{1} .
$$

In the Hilbert case, Theorem 4.6 can be found in [22].

Acknowledgements. It is a pleasure to thank Professor Hans Triebel for some helpful discussions during the preparation of the paper. Some of them happened in the unique atmosphere of the Mathematisches Forschungsinstitut Oberwolfach during the Mini-Workshop "Compactness Problems in Interpolation Theory and Function Spaces" (August 15-21, 2004). We would like also to express our gratitude to the MFO. 


\section{References}

[1] Arazy, J., Fisher, S. D. and Peetre, J., Hankel operators on weighted Bergman spaces. Amer. J. Math. 110 (1988), 989 - 1053.

[2] Bennett, C. and Rudnick, K., On Lorentz-Zygmund spaces. Warsaw: Polish Acad. Sci. Inst. Math., Dissertationes Math. 175 (1980).

[3] Bennett, C. and Sharpley, R., Interpolation of Operators. Boston: Academic Press 1988.

[4] Bergh, J. and Löfström, J., Interpolation Spaces. An Introduction. Berlin: Springer 1976.

[5] Cobos, F., On the Lorentz-Marcinkiewicz operator ideal. Math. Nachr. 126 (1986), $281-300$.

[6] Cobos, F., Entropy and Lorentz-Marcinkiewicz operator ideals. Arkiv Mat. 25 (1987), $211-219$.

[7] Cobos, F., Fernández-Cabrera, L. M. and Triebel, H., Abstract and concrete logarithmic interpolation spaces. J. London Math. Soc. 70 (2004), 231 - 243.

[8] Edmunds, D. E. and Evans, W. D., Hardy Operators, Function Spaces and Embeddings. Heidelberg: Springer 2004.

[9] Edmunds, D. E. and Triebel, H., Logarithmic Sobolev spaces and their applications to spectral theory. Proc. London Math. Soc. 71 (1995), 333 - 371.

[10] Edmunds, D. E. and Triebel, H., Function Spaces, Entropy Numbers, Differential Operators. Cambridge: Cambridge University Press 1996.

[11] Edmunds, D. E. and Triebel, H., Logarithmic spaces and related trace problems. Funct. Approx. Comment. Math. 26 (1998), 189 - 204.

[12] Fernández-Cabrera, L. M., Cobos, F., Hernández, F. L. and Sánchez, V. M., Indices defined by interpolation scales and applications. Proc. Royal Soc. Edinburgh 134A (2004), $695-717$.

[13] Fiorenza, A. and Karadzhov, G. E., Grand and small Lebesgue spaces and their analogs. Z. Anal. Anwendungen 23 (2004), $657-681$.

[14] Gohberg, I. C. and Krein, M. G., Introduction to the Theory of Linear Nonselfadjoint Operators (transl. from Russian). Providence, R.I.: Amer. Math. Soc. 1969.

[15] Gohberg, I. C. and Krein, M. G., Theory and Applications of Volterra Operators in Hilbert Space (transl. from Russian). Providence, R.I.: Amer. Math. Soc. 1970.

[16] Gustavsson, J., A function parameter in connection with interpolation of Banach spaces. Math. Scand. 42 (1978), $289-305$.

[17] Janson, S., Minimal and maximal methods of interpolation. J. Funct. Anal. 44 (1981), $50-73$.

[18] Jawerth, B. and Milman, M., Extrapolation Theory with Applications. Providence, R.I.: Mem. Amer. Math. Soc. 89 (1991), no. 440. 
[19] Karadzhov, G. E. and Milman, M., Extrapolation theory: new results and applications. J. Approx. Theory 133 (2005), 38 - 99.

[20] König, H., Interpolation of operator ideals with an application to eigenvalue distribution problems. Math. Ann. 233 (1978), 35 - 48.

[21] König, H., Eigenvalue Distribution of Compact Operators. Basel: Birkhäuser 1986.

[22] Milman, M., Extrapolation and Optimal Decompositions. Lecture Notes in Mathematics 1580. Berlin: Springer 1994.

[23] Opic, B. and Pick, L., On generalized Lorentz-Zygmund spaces. Math. Inequal. Appl. 2 (1999), 391 - 467.

[24] Peetre, J., A Theory of Interpolation of Normed Spaces. Notas Mat. 39 (Lectures Notes, Brasilia, 1963). Rio de Janeiro: Inst. Mat. Pura Apl. 1968.

[25] Persson, L.-E., Interpolation with a parameter function. Math. Scand. 59 (1986), $199-222$.

[26] Pietsch, A., Operator Ideals. Amsterdam: North-Holland 1980

[27] Pietsch, A., Eigenvalues and s-Numbers. Cambridge: Cambridge University Press 1987.

[28] Stein, E. M., Singular Integrals and Differentiability Properties of Functions. Princeton, N.J.: Princeton Univ. Press 1970.

[29] Strichartz, R. S., A note on Trudinger's extension of Sobolev's inequality. Indiana Univ. Math. J. 21 (1972), $841-842$.

[30] Triebel, H., Interpolation Theory, Function Spaces, Differential Operators. Amsterdam: North-Holland 1978 (sec. ed. Leipzig: Barth 1995.)

[31] Triebel, H., Approximation numbers and entropy numbers of embeddings of fractional Besov-Sobolev spaces in Orlicz spaces. Proc. London Math. Soc. 66 (1993), $589-618$.

[32] Triebel, H., Fractals and Spectra Related to Fourier Analysis and Function Spaces. Basel: Birkhäuser 1997.

[33] Trudinger, N. S., On imbeddings into Orlicz spaces and some applications. J. Math. Mech. 17 (1967), 473 - 483.

Received June 6, 2005 